\title{
Microstrip Patch Antenna Array for a Scalable X-band Radar System
}

\author{
Steven D. Keller*, Steven Weiss \\ U.S. Army Research Laboratory, Adelphi, MD 20783, USA \\ Email: steven.keller4@us.army.mil
}

\section{Introduction}

The enhancement of situational awareness at the unit level is critical for ensuring optimal security and battlefield performance for the warfighter. Most state-of-the-art military radar systems are too large and high-cost to be available to each individual unit or to high-volume fleets of small unmanned vehicles and sensors. In an effort to improve situational awareness at the unit level, research and development is underway to demonstrate a scalable radar system of appropriate size, weight and power (SWAP) for persistent surveillance. The scalability of the technology will facilitate the development of a suite of radars for a variety of applications and platforms, including unattended ground sensors (UGS), unmanned systems, and man-portable sensors.

A 32-element X-band microstrip electronic scanning array (ESA) has been developed to serve as the radiating element in a preliminary system radar testbed. An array of microstrip aperture coupled patch antennas [1] are fed equal power through a Wilkinson power divider network and the direction of radiation is controlled by surface mount phase shifters integrated into the RF control board. While bulky physical array steering mounts greatly impact the system weight and visual signature of traditional radar systems, the electronic beam steering mechanism employed in this system minimizes each of these attributes. The details of the antenna array element in this radar ESA design will be examined in the subsequent sections.

\section{Patch Antenna Design}

The radar testbed under development requires a specific X-band center frequency (referred to as , $\mathrm{f}_{\mathrm{c}}^{\text {'e }}$ for the remainder of this paper), $0.8 \%$ bandwidth, and $90^{\circ}$ scan angle with a $9^{\circ}$ beamwidth in azimuth and $18^{\circ}$ beamwidth in elevation for optimal functionality. In order to satisfy these specifications, a $4 \times 8$ patch antenna array was designed using a microstrip aperture coupled feed technique.

As a first step, a single patch antenna was designed to operate on 62 mil thick RT/Duroid $5870\left(\varepsilon_{\mathrm{r}}=2.33\right)$ with a microstrip feedline layer constructed on a bottom layer of $20 \mathrm{mil}$ thick RT/Duroid $3010\left(\varepsilon_{\mathrm{r}}=10.2\right)$, as shown in Figure 1. The thin, high dielectric feedline substrate ensured that the field lines from the microstrip feedlines would be well contained and consequently minimized undesirable feedline radiation. The thick, low dielectric substrate for the patch layer was strategically employed to maximize the usable bandwidth range and radiated power and to minimize dielectric, conductor and surface wave losses. The initial design for the microstrip patch antenna has patch dimensions of $\sim 0.414 \lambda_{\mathrm{RT} / \mathrm{D} 5870}$ length and $0.473 \lambda_{\mathrm{RT} / \mathrm{D} 5870}$ width, microstrip feedline width of $\sim 0.041 \lambda_{\mathrm{RT} / \mathrm{D} 3010}$, ground plane slot size of $0.22 \lambda_{\mathrm{RT} / \mathrm{D} 5870}$ length and $\sim 0.026 \lambda_{\mathrm{RT} / \mathrm{D} 5870}$ width, and tuning stub length of $0.156 \lambda_{\mathrm{RT} / \mathrm{D} 3010}$, where $\lambda_{\mathrm{RT} / \mathrm{D} 5870}$ is the wavelength in the $\mathrm{RT} /$ Duroid 5870 substrate and $\lambda_{\mathrm{RT} / \mathrm{D} 3010}$ is the wavelength in the RT/Duroid 3010 substrate. 
The 2.5-D (layered 2-D) simulator EMPiCASSO [2] was used to design the patch antenna element and optimize the antenna array for functionality at desired center frequency. The Method of Moments code employed in EMPiCASSO makes it exceptionally efficient for optimizing planar antenna structures such as the aperture coupled patch design. Optimization techniques for aperture coupled patch antenna designs detailed in [3] were particularly useful in tweaking the original patch design to overcome mutual coupling effects between elements in the antenna array.
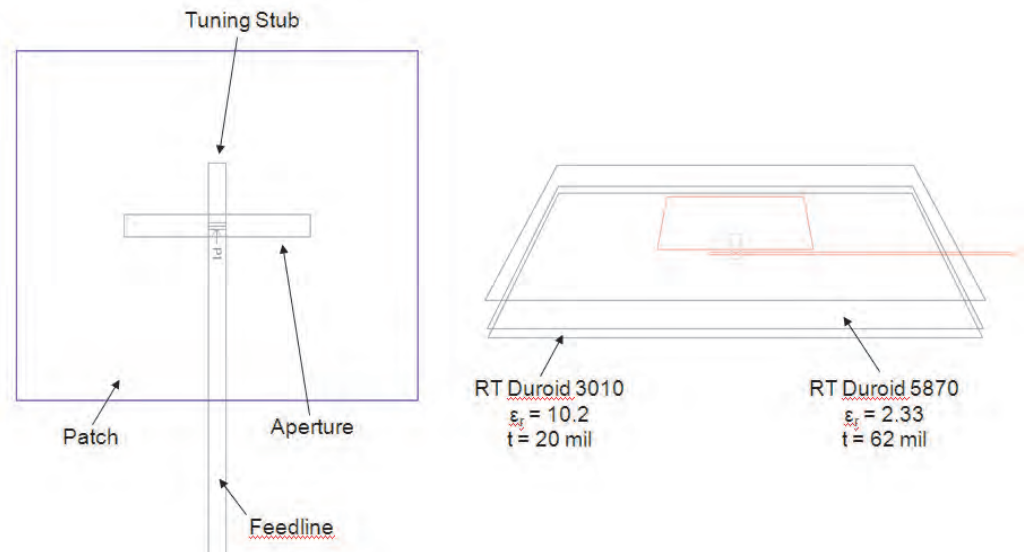

Figure 1. EMPiCASSO model of X-band microstrip slot-coupled patch antenna.

The antenna design was simulated in EMPiCASSO to optimize the center frequency and bandwidth and verify a broadside radiation pattern with acceptable gain. The reflection coefficient plot shown in Figure 2 indicates an excellent resonance just below the desired center frequency with a $-10 \mathrm{~dB} \mathrm{~S}_{11}$ bandwidth of $\sim 7.4 \%$. While this simulated patch center frequency is approximately $2.6 \%$ lower than the desired operational frequency of the full antenna array, the microstrip feedline network and mutual coupling effects between antenna elements that arise when the patch is put into a $4 \times 8$ array will impact this center frequency. The simulation results detailed in the next section will illustrate this effect.

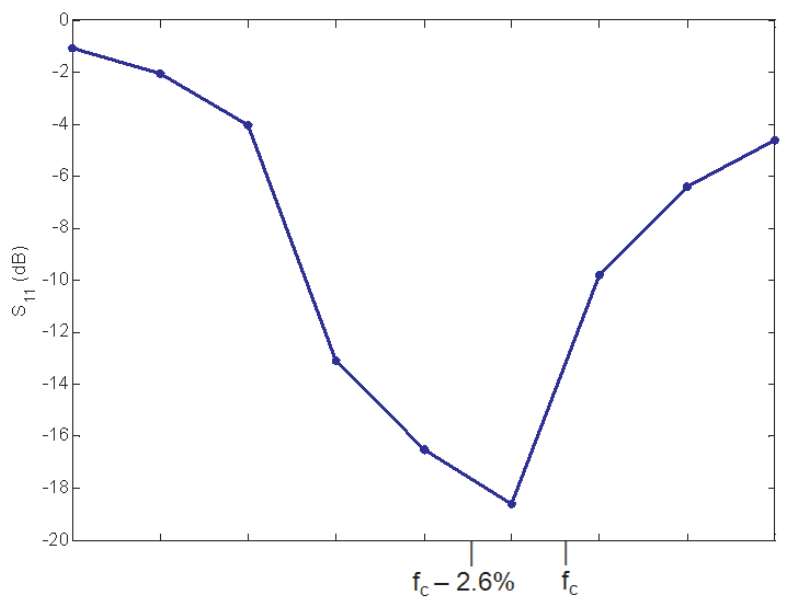

Figure 2. Simulated reflection coefficient for patch antenna. 


\section{Antenna Array Design}

Having optimized a patch antenna to serve as the array element, a model of the antenna array with a full microstrip feedline network was constructed in EMPiCASSO. In order to produce equal power division between the antennas, a Wilkinson power divider strategy [4] was employed with $70 \Omega$ quarter wavelength microstrip lines and a shunt $100 \Omega$ chip resistor at the $50 \Omega$ line division, as shown in Figure 3. Due to program memory constraints, the simulation was limited to a $4 \times 3$ element array. However, the significant mutual coupling effects from arraying the patch elements should be reasonably accounted for by this simulation since most of the coupling should result from the E-plane elements and all of these elements (4 patches) are included in the simulation model.

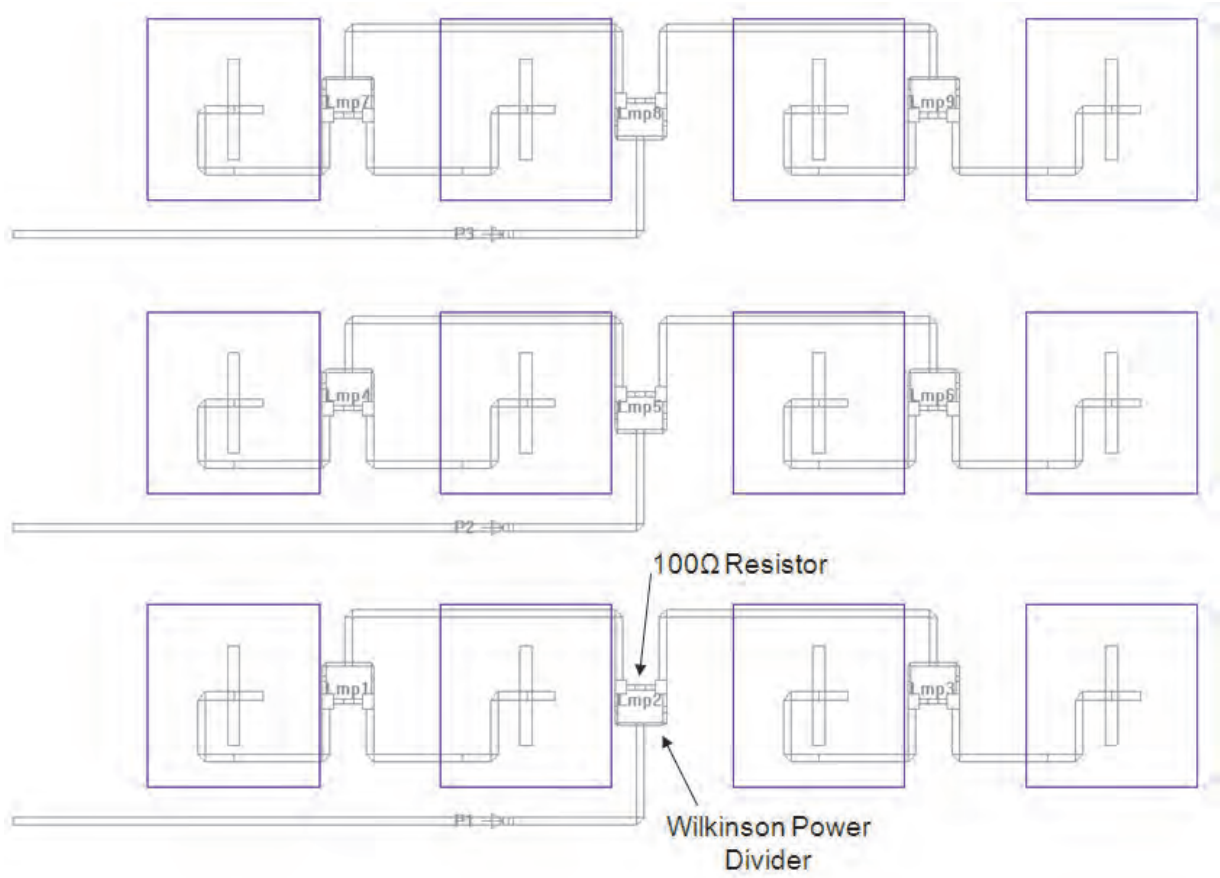

Figure 3. EMPiCASSO model of X-band microstrip slot-coupled patch antenna array.

The simulated reflection coefficients for each row of elements in this array are shown in Figure 4 as „Old $\mathrm{S}_{11}{ }^{\text {"e }}$, Old $\mathrm{S}_{22}{ }^{{ }^{e}}$, and „Old $\mathrm{S}_{33}{ }^{e}$. The mutual coupling between elements and feedline network resulted in a detuned design with the center frequency shifted to approximately $3.3 \%$ above the desired center frequency and the $-10 \mathrm{~dB} \mathrm{~S} \mathrm{~S}_{11}$ bandwidth decreased to $\sim 1 \%$. With these results and the parametric optimization tools of EMPiCASSO, the dimensions of each patch antenna were adjusted to yield an optimal resonance at the desired center frequency. The patch length was increased by $8 \%$ and the ground plane slot length and width were both increased $\sim 20 \%$. All other design detailed remained unchanged. The optimized reflection coefficients for each row of array elements is shown in Figure 4 as „New $\mathrm{S}_{11}{ }^{e}$, „New $\mathrm{S}_{2}{ }^{e}$, and „New $\mathrm{S}_{3}{ }^{{ }^{e e}}$ and yields a center frequency extremely close to the desired center frequency and more than sufficient bandwidth of $2.6 \%$. This design should satisfy the necessary center frequency and bandwidth specifications for the X-band radar testbed. 


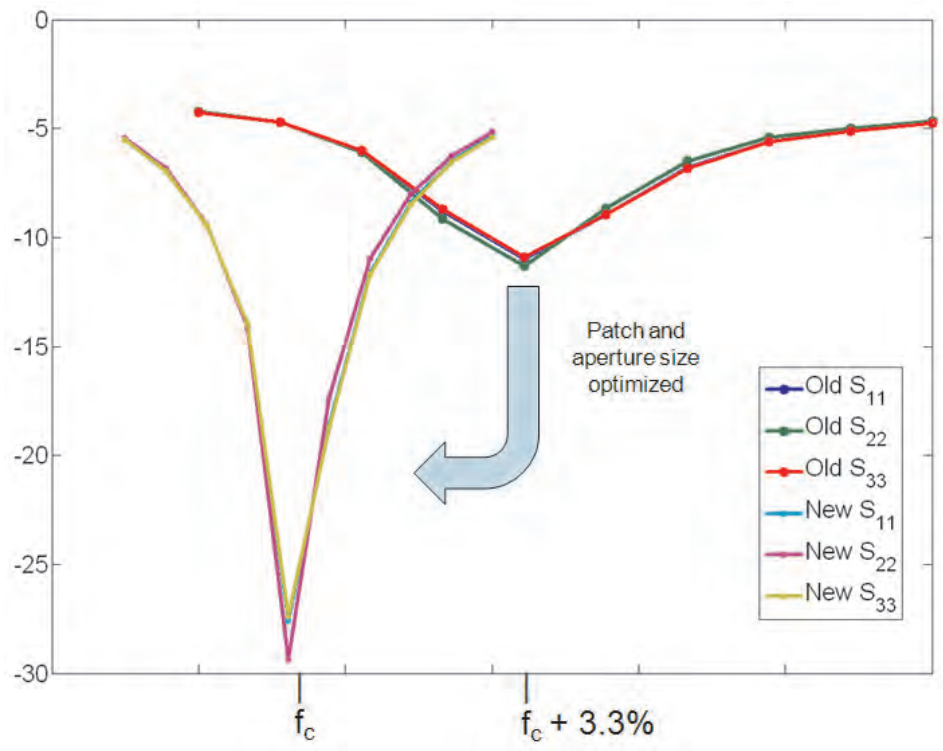

Figure 4. Optimized reflection coefficient for X-band patch antenna array.

\section{Conclusion}

The design of an X-band microstrip aperture coupled patch antenna array to facilitate electronic scanning in a scalable radar testbed has been described above. The measured reflection coefficient, radiation pattern and gain measurements of the prototype antenna and beam steering demonstration of the device connected with the full RF control board will be presented at the 2010 IEEE Antennas and Propagation Society Conference.

\section{References}

[1] D. M. Pozar, "Microstrip antenna aperture-coupled to a microstrip line", Electron. Lett., vol. 21, no. 2, pp. 49-50, January 1985.

[2] EMAG Technologies Inc, EMPiCASSO, http://www.emagware.com/empicasso.html, 2009.

[3] P. L. Sullivan and D. H. Schaubert, "Analysis of an aperture coupled microstrip antenna", IEEE Transactions on Antennas and Propagation, vol. 34, no. 8, pp. 977984, August 1986.

[4] D. Pozar, Microwave engineering, pp. 318-323, John Wiley \& Sons, 3rd edition, 2005. 